\title{
Visual Disability in Mexico
}

\author{
Roberto Cruz Gonzalez* \\ Professor of Ophthalmology, Faculty of Medicine, Mexico
}

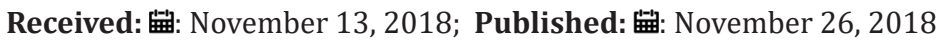

*Corresponding author: Roberto Cruz Gonzalez, Professor of Ophthalmology, Faculty of Medicine and Health Sciences, Mexico

\section{Opinion}

Knowing what level of health, the population of a country has an indicator of wealth, prosperity and well-being. With the population growth and the increase in life expectancy, also with an increase in chronic degenerative diseases, a greater active participation of society in these health problems is required. Disability due to visual diseases is one of the public health problems that is present now days and is taking high importance in the world, this problem presents a big impact in the economy of families and countries in the total population due to its rapidly growing. It is known that visual disabilities $90 \%$ occur in developing countries with low incomes and poor health systems and it is also known that $80 \%$ of cases of visual disability can be prevented according to the Global Data on visual impairments 2010, World Health Organization 2012 (who/nmh/pbd/12.01).

Mexico has become one of the most populated countries in America with approximately 120 million habitants. Currently $27 \%$ of the population is under 15 years and $11 \%$ over 60 years. By 2050 , a growth of $22 \%$ in the population is expected. By then population will increase 45 million, $30 \%$ of Mexicans will be over 60 years with an expectation of life of 83 years. The country will face greater visual health challenges than the current ones, with a society that grows and ages in a country of contrasts where inequality in access to education and health services is increasing the challenge of having a good health programs will increase as well creating a virtuous circle. In 2013, the World Health Assembly approved the World Plan of Action 2014-2019 “Universal Eye Health: 2014-2019 Global Plan of Action." During the 66th World Health Assembly A66/11, March 28, 2013 World Health Organization. The vision of the Plan is to envision a world in which no one suffers from visual disability due to avoidable causes and where people with unavoidable vision loss can reach their full potential by strengthening access to comprehensive ophthalmic care services.

In 2014, with great enthusiasm and interest, they motivated a group of Mexican Ophthalmologists with training in Public Health who decided to join and found the Mexican Center of Preventive Visual Health, A.C. (CMSVP). With the intention of joining the efforts made by the Ministry of Health of Mexico in implementing programs through the National Council for the Prevention and Treatment of Visual Diseases, an area created in 2003 by the federal government with minimal impact on achievements in visual health. Currently the CMSVP is recognized as a High Specialty group of the Mexican Society of Ophthalmology. The mission of the CMSVP is to reduce the prevalence of blindness and avoidable visual disability in Mexico as a challenge in Public Health. The Vision is to live in a Mexico that has a comprehensive system of efficient, accessible and quality visual health care for all, avoiding blindness and visual disability. The CMSVP has identified the main visual health problems that affect Mexicans, such as cataracts, diabetic retinopathy, age-related macular degeneration, glaucoma, retinopathy of prematurity and refractive errors.

Public Health must be get closer to therapeutic medicine, not only focus on treating the disease, but to see and detect the external factors and the environment that causes the visual problem. Public Health invites us to a different culture, we must transmit and educate the population, so they are better informed and better situated in objective reality in the same way prepare medical students, doctors, health professionals and ophthalmologists in training who will be responsible for continuing, improving, transforming or discarding these preventive visual health programs. The actions of the CMSVP are to promote awareness of prevention, early detection and rehabilitation of visual diseases at all levels of medical care. We know that investing in prevention programs benefits the population and generates economic development for them, for their families and for the country. We are interested in collaboration with Educational Institutions, Civil Society, Pharmaceutical Industry, Government, National and International Non-Governmental Organizations to share knowledge, experience and tools that are useful in the generation of public policies that improve visual health and prevent their disability. 
ISSN: 2574-1241

DOI: 10.26717/BJSTR.2018.11.002099

Roberto Cruz Gonzalez. Biomed J Sci \& Tech Res

(C) (P) This work is licensed under Creative

Submission Link: https://biomedres.us/submit-manuscript.php

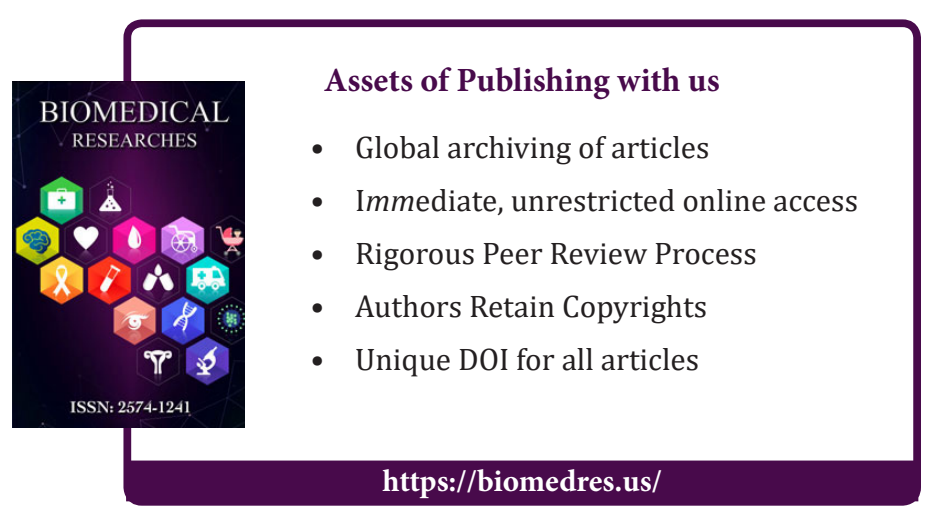

